\title{
ON THE GLOBAL EXISTENCE FOR A REGULARIZED MODEL OF VISCOELASTIC NON-NEWTONIAN FLUID
}

\author{
ONDŘEJ KREML, MILAN POKORNÝ AND PAVEL ŠALOM
}

\begin{abstract}
We study the generalized Oldroyd model with viscosity depending on the shear stress behaving like $\mu(\mathbf{D}) \sim|\mathbf{D}|^{p-2}\left(p>\frac{6}{5}\right)$ regularized by a nonlinear stress diffusion. Using the Lipschitz truncation method we are able to prove global existence of weak solution to the corresponding system of partial differential equations.
\end{abstract}

\section{INTRODUCTION}

The well known Oldroyd model describing the flow of incompressible viscoelastic fluid consists of the following system of partial differential equations

$$
\begin{aligned}
\operatorname{div} \mathbf{v} & =0 \\
\partial_{t} \mathbf{v}+\operatorname{div}(\mathbf{v} \otimes \mathbf{v}) & =-\nabla \pi+\mu \Delta \mathbf{v}+\operatorname{div} \mathbf{T}, \\
\mathbf{T}+\partial_{t} \mathbf{T}+\mathbf{v} \cdot \nabla \mathbf{T} & =2 \mu_{0} \mathbf{D}+\mathbf{W} \mathbf{T}-\mathbf{T} \mathbf{W}+a(\mathbf{D T}+\mathbf{T D}) .
\end{aligned}
$$

Here the unknowns are the velocity vector $\mathbf{v}$, the pressure $\pi$ and the symmetric extra stress tensor $\mathbf{T}$. The tensor $\mathbf{D}$ denotes the symmetric part of the velocity gradient $\mathbf{D}=\mathbf{D}(\mathbf{v})=$ $\frac{1}{2}\left(\nabla \mathbf{v}+(\nabla \mathbf{v})^{T}\right)$ and $\mathbf{W}$ denotes its skew-symmetric part $\mathbf{W}=\mathbf{W}(\mathbf{v})=\frac{1}{2}\left(\nabla \mathbf{v}-(\nabla \mathbf{v})^{T}\right)$, $\mu$ and $\mu_{0}$ are positive constants and $a \in[-1,1]$ is a real parameter. Special choices $a=-1,0,1$ yield respectively the lower convected (Oldroyd A), corotational and upper convected (Oldroyd B) models.

With the exception of the work of Lions and Masmoudi [10, where the authors proved global existence of weak solutions for the corotational model, the global existence theory for the Oldroyd models is still an open problem. Existence of weak solutions to (1.1) for general $a$ is proved only under some smallness assumptions, either on the time interval or the initial data (see e.g. [5, 8, 9], [11).

It is well known that some fluids as e.g. the blood exhibit both the viscoelastic and shearthinning behavior. Therefore it is important to consider models which can describe these properties. In this paper we propose a generalized, and regularized, version of the Oldroyd system (1.1). Namely, instead of a constant viscosity coefficient $\mu$ in $(1.1)_{2}$ we introduce shear dependent viscosity $\mu(\mathbf{D})$ with properties specified later. This enables the model to describe better the shear thinning behavior of the fluid (or shear thickening, if needed). As even the model with constant viscosity is (except for a special case discussed above) not

Key words and phrases. viscoelastic fluid; non-Newtonian fluid; Lipschitz truncation; global existence.

O.K. acknowledges the support of the GAČR (Czech Science Foundation) project GA13-00522S in the general framework of RVO: 67985840 . 
known to posses a weak solution, the least it can be expected for a more complex (and less regular) model. Hence we regularize equation (1.1) 3 for the extra stress by introducing a (nonlinear) stress diffusion. Denoting

$$
\mathbf{B}(\mathbf{v}, \mathbf{T}):=\mathbf{W} \mathbf{T}-\mathbf{T W}+a(\mathbf{D T}+\mathbf{T D})
$$

the system we study is the following

$$
\begin{aligned}
\operatorname{div} \mathbf{v} & =0 \\
\partial_{t} \mathbf{v}+\operatorname{div}(\mathbf{v} \otimes \mathbf{v})+\nabla \pi-\operatorname{div}(\mu(\mathbf{D}) \mathbf{D}) & =\operatorname{div} \mathbf{T} \\
\partial_{t} \mathbf{T}+\mathbf{v} \cdot \nabla \mathbf{T}-\varepsilon \operatorname{div}(\gamma(\nabla \mathbf{T}) \nabla \mathbf{T})+\mathbf{T} & =2 \mu_{0} \mathbf{D}+\mathbf{B}(\mathbf{v}, \mathbf{T}) .
\end{aligned}
$$

Here $\varepsilon$ is a positive constant and the properties of functions $\mu(\mathbf{D})$ and $\gamma(\nabla \mathbf{T})$ are stated later.

We consider our system (1.3) on a space-time cylinder $\Omega \times[0, T)$ where $\Omega \subset \mathbb{R}^{3}$ is a bounded domain with Lipschitz boundary and we add the initial conditions

$$
\begin{aligned}
& \mathbf{v}(\mathbf{x}, 0)=\mathbf{v}_{0}(\mathbf{x}) \quad \text { in } \Omega, \\
& \mathbf{T}(\mathbf{x}, 0)=\mathbf{T}_{0}(\mathbf{x}) \quad \text { in } \Omega
\end{aligned}
$$

and the boundary conditions

$$
\begin{aligned}
\mathbf{v}=\mathbf{0} & \text { on } \partial \Omega \times[0, T), \\
\frac{\partial \mathbf{T}}{\partial \mathbf{n}}=\mathbf{0} & \text { on } \partial \Omega \times[0, T) .
\end{aligned}
$$

We assume that the function $\mu: \mathbb{R}^{3 \times 3} \rightarrow \mathbb{R}^{+}$satisfies the following conditions. For some $p>1$

(i) $\mu(\mathbf{D}) \mathbf{D}$ is $p$-coercive, i.e.

$$
\exists c>0 \exists \varphi_{1} \in L^{1}(\Omega \times(0, T)) \forall \mathbf{D} \in \mathbb{R}_{\mathrm{sym}}^{3 \times 3}: \quad \mu(\mathbf{D})|\mathbf{D}|^{2} \geq c|\mathbf{D}|^{p}-\varphi_{1},
$$

(ii) $\mu(\mathbf{D}) \mathbf{D}$ has $(p-1)$-growth, i.e.

$$
\exists c>0 \exists \varphi_{2} \in L^{p^{\prime}}(\Omega \times(0, T)) \forall \mathbf{D} \in \mathbb{R}_{\mathrm{sym}}^{3 \times 3}: \quad \mu(\mathbf{D})|\mathbf{D}| \leq c|\mathbf{D}|^{p-1}+\varphi_{2},
$$

(iii) $\mu(\mathbf{D}) \mathbf{D}$ is strictly monotone, i.e.

$$
\forall \mathbf{D}_{1}, \mathbf{D}_{2} \in \mathbb{R}_{\text {sym }}^{3 \times 3}, \mathbf{D}_{1} \neq \mathbf{D}_{2}: \quad\left(\mu\left(\mathbf{D}_{1}\right) \mathbf{D}_{1}-\mu\left(\mathbf{D}_{2}\right) \mathbf{D}_{2}\right):\left(\mathbf{D}_{1}-\mathbf{D}_{2}\right)>0,
$$

and similarly $\gamma: \mathbb{R}^{3 \times 3 \times 3} \rightarrow \mathbb{R}^{+}$satisfies for some $q>1$

(iv) $\gamma(\nabla \mathbf{T}) \nabla \mathbf{T}$ is $q$-coercive, i.e.

$$
\exists c>0 \exists \varphi_{3} \in L^{1}(\Omega \times(0, T)) \forall \mathbf{T} \in \mathbb{R}_{\mathrm{sym}}^{3 \times 3}: \quad \gamma(\nabla \mathbf{T})|\nabla \mathbf{T}|^{2} \geq c|\nabla \mathbf{T}|^{q}-\varphi_{3},
$$

(v) $\gamma(\nabla \mathbf{T}) \nabla \mathbf{T}$ has $(q-1)$-growth, i.e.

$$
\exists c>0 \exists \varphi_{4} \in L^{q^{\prime}}(\Omega \times(0, T)) \forall \mathbf{T} \in \mathbb{R}_{\mathrm{sym}}^{3 \times 3}: \quad \gamma(\nabla \mathbf{T})|\nabla \mathbf{T}| \leq c \mid \nabla \mathbf{T}^{q-1}+\varphi_{4},
$$

(vi) $\gamma(\nabla \mathbf{T}) \nabla \mathbf{T}$ is monotone, i.e.

$$
\forall \mathbf{T}_{1}, \mathbf{T}_{2} \in \mathbb{R}_{\text {sym }}^{3 \times 3}: \quad\left(\gamma\left(\nabla \mathbf{T}_{1}\right) \nabla \mathbf{T}_{1}-\gamma\left(\nabla \mathbf{T}_{2}\right) \nabla \mathbf{T}_{2}\right):\left(\nabla \mathbf{T}_{1}-\nabla \mathbf{T}_{2}\right) \geq 0 .
$$


This model was first introduced in [7] where existence of weak solutions for $p>\frac{8}{5}$ and $q$ sufficiently large was proven for the problem with either periodic boundary conditions or complete slip boundary conditions for the velocity $\mathbf{v}$. The proof in [7] is based on the $L^{\infty}$ test functions technique developed by Frehse, Málek and Steinhauer in [6]. The case of Dirichlet boundary conditions for problem (1.3) was studied in [12], where existence of weak solutions is proved for $p>\frac{6}{5}$ and $q>2 p^{\prime}$. The proof is based on the Lipschitz truncation method and the construction of local pressure from [4]. Moreover, in both cases, additional lower order nonlinear term was used in order to obtain suitable a-priori estimates. In this paper we further improve the condition on $q$, under which the existence of weak solutions is proved, remove the additional lower-order term and based on the recent result for the Lipschitz truncation to the solenoidal functions (see [1]) we also significantly shorten the proof.

Similar model was studied in 2] where the authors consider classical Oldroyd-B model with constant viscosity $\mu$ and linear stress diffusion and prove global regularity of solutions in $2 \mathrm{D}$.

In the whole text we denote vectors by small bold letters and tensors by capital bold letters. We introduce the following function spaces.

$$
\begin{aligned}
& \mathcal{C}_{0, \text { div }}^{\infty}(\Omega):=\left\{\mathbf{v}: \mathbb{R}^{3} \rightarrow \mathbb{R}^{3} \mid \mathbf{v} \in \mathcal{C}_{0}^{\infty}(\Omega), \operatorname{div} \mathbf{v}=0\right\},
\end{aligned}
$$

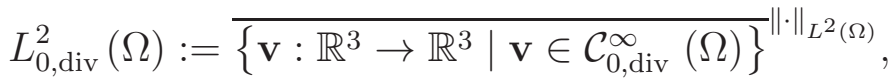

$$
\begin{aligned}
& W_{0, \operatorname{div}}^{1, p}(\Omega):=\overline{\left\{\mathbf{v}: \mathbb{R}^{3} \rightarrow \mathbb{R}^{3} \mid \mathbf{v} \in \mathcal{C}_{0, \operatorname{div}}^{\infty}(\Omega)\right\}} \|^{\|\cdot\|_{W^{1, p}(\Omega)}}, \\
& L_{\text {sym }}^{2}(\Omega):=\overline{\left\{\mathbf{T}: \mathbb{R}^{3} \rightarrow \mathbb{R}^{3 \times 3} \mid \mathbf{T} \in \mathcal{C}^{\infty}(\bar{\Omega}), \mathbf{T} \text { symmetric }\right\}}\|\cdot\|_{L^{2}(\Omega)}, \\
& W_{\text {sym }}^{1, q}(\Omega):=\overline{\left\{\mathbf{T}: \mathbb{R}^{3} \rightarrow \mathbb{R}^{3 \times 3} \mid \mathbf{T} \in \mathcal{C}^{\infty}(\bar{\Omega}), \mathbf{T} \text { symmetric }\right\}}{ }^{\|\cdot\|_{W^{1, q}(\Omega)}} .
\end{aligned}
$$

Moreover, we denote $X^{*}$ the dual space to $X$ and by $\langle T, \varphi\rangle_{k, r}$ we mean duality between spaces $W^{k, r}(\Omega)$ and $\left(W^{k, r}(\Omega)\right)^{*}$, similarly for duality between Sobolev space of solenoidal functions with zero trace (or symmetric tensors) and its dual we use $\langle T, \varphi\rangle_{k, r \text {,div }}$ (or $\langle T, \varphi\rangle_{k, r \text {,sym }}$ respectively). For $t>0$ we denote $Q_{t}=\Omega \times(0, t)$ the space-time cylinder. For $s \in[1, \infty]$ we denote $s^{\prime}$ its dual exponent, i.e. $\frac{1}{s}+\frac{1}{s^{\prime}}=1$.

Before defining weak solution of (1.3) we denote

$$
\mathbf{A}(\mathbf{T}, \boldsymbol{\psi})=-\mathbf{T} \boldsymbol{\psi}+(\mathbf{T} \boldsymbol{\psi})^{T}+a\left(\mathbf{T} \boldsymbol{\psi}+(\mathbf{T} \boldsymbol{\psi})^{T}\right)
$$

and observe that integrating by parts and using the boundary condition (1.5) $)_{1}$ it holds

$$
\int_{\Omega} \mathbf{B}(\mathbf{v}, \mathbf{T}): \boldsymbol{\psi} \mathrm{d} \mathbf{x} \mathrm{d} t=-\int_{\Omega} \mathbf{v} \cdot \operatorname{div} \mathbf{A}(\mathbf{T}, \boldsymbol{\psi}) \mathrm{d} \mathbf{x} \mathrm{d} t
$$

for all $\boldsymbol{\psi} \in \mathcal{C}^{\infty}(\bar{\Omega})$. Note moreover that in our case $\boldsymbol{\psi}$ and $\mathrm{T}$ are symmetric, therefore

$$
\mathbf{A}(\mathbf{T}, \boldsymbol{\psi})=\boldsymbol{\psi} \mathbf{T}-\mathbf{T} \boldsymbol{\psi}+a(\mathbf{T} \boldsymbol{\psi}+\boldsymbol{\psi} \mathbf{T}) .
$$


Definition 1.1. Let $\mathbf{v}_{0} \in L_{0 \text {,div }}^{2}(\Omega), \mathbf{T}_{0} \in L_{\text {sym }}^{2}(\Omega)$ and let $\varepsilon, \mu_{0}, T$ be positive constants. Let $\mu: \mathbb{R}^{3 \times 3} \rightarrow \mathbb{R}^{+}$be a continuous function satisfying (1.6)-(1.8) with some $p>\frac{6}{5}$ and let $\gamma: \mathbb{R}^{3 \times 3 \times 3} \rightarrow \mathbb{R}^{+}$be a continuous function satisfying (1.9)-(1.11) with some $q>1$. We say that a couple $(\mathbf{v}, \mathbf{T})$ is a weak solution of system (1.3) with initial conditions (1.4) and boundary conditions (1.5) if

$$
\begin{aligned}
\mathbf{v} & \in L^{\infty}\left(0, T ; L_{0, \operatorname{div}}^{2}(\Omega)\right) \cap L^{p}\left(0, T ; W_{0, \operatorname{div}}^{1, p}(\Omega)\right), \\
\mathbf{T} & \in L^{\infty}\left(0, T ; L_{\text {sym }}^{2}(\Omega)\right) \cap L^{q}\left(0, T ; W_{\text {sym }}^{1, q}(\Omega)\right), \\
\partial_{t} \mathbf{v} & \in L^{\sigma}\left(0, T ;\left(W_{0, \operatorname{div}}^{1, \sigma^{\prime}}(\Omega)\right)^{*}\right), \quad \text { for some } \quad 1 \leq \sigma \leq \frac{5}{6} p, \\
\partial_{t} \mathbf{T} & \in L^{q^{\prime}}\left(0, T ;\left(W_{\mathrm{sym}}^{1, q}(\Omega)\right)^{*}\right)
\end{aligned}
$$

and for almost all $t \in(0, T)$ it holds

$$
\left\langle\partial_{t} \mathbf{v}(t), \boldsymbol{\varphi}\right\rangle_{1, \sigma^{\prime}, \mathrm{div}}+\int_{\Omega}(\mu(\mathbf{D}(t)) \mathbf{D}(t)-(\mathbf{v} \otimes \mathbf{v})): \nabla \boldsymbol{\varphi} \mathrm{d} \mathbf{x}=\int_{\Omega} \operatorname{div} \mathbf{T}(t) \cdot \boldsymbol{\varphi} \mathrm{d} \mathbf{x}
$$

for all $\varphi \in \mathcal{C}_{0, \operatorname{div}}^{\infty}(\Omega)$,

$$
\begin{array}{r}
\left\langle\partial_{t} \mathbf{T}(t), \boldsymbol{\psi}\right\rangle_{1, q, \text { sym }}+\int_{\Omega}(\mathbf{v}(t) \cdot \nabla \mathbf{T}(t)): \boldsymbol{\psi} \mathrm{d} \mathbf{x} \\
+\varepsilon \int_{\Omega} \gamma(\nabla \mathbf{T}(t)) \nabla \mathbf{T}(t): \nabla \boldsymbol{\psi} \mathrm{d} \mathbf{x}+\int_{\Omega} \mathbf{T}: \boldsymbol{\psi} \mathrm{d} \mathbf{x} \\
=2 \mu_{0} \int_{\Omega} \mathbf{D}(t): \boldsymbol{\psi} \mathrm{d} \mathbf{x}-\int_{\Omega} \mathbf{v}(t) \cdot \operatorname{div} \mathbf{A}(\mathbf{T}(t), \boldsymbol{\psi}) \mathrm{d} \mathbf{x}
\end{array}
$$

for all $\boldsymbol{\psi} \in \mathcal{C}^{\infty}(\bar{\Omega}), \boldsymbol{\psi}$ symmetric,

$$
\begin{aligned}
& \forall \boldsymbol{\varphi} \in \mathcal{C}_{0, \mathrm{div}}^{\infty}(\Omega): \quad \lim _{t \rightarrow 0_{+}} \int_{\Omega} \mathbf{v}(t) \cdot \boldsymbol{\varphi} \mathrm{d} \mathbf{x}=\int_{\Omega} \mathbf{v}_{0} \cdot \boldsymbol{\varphi} \mathrm{d} \mathbf{x} \\
& \forall \boldsymbol{\psi} \in \mathcal{C}^{\infty}(\bar{\Omega}): \quad \lim _{t \rightarrow 0_{+}} \int_{\Omega} \mathbf{T}(t): \boldsymbol{\psi} \mathrm{d} \mathbf{x}=\int_{\Omega} \mathbf{T}_{0}: \boldsymbol{\psi} \mathrm{d} \mathbf{x}
\end{aligned}
$$

and $(1.5)_{1}$ is fulfilled for almost all $t \in(0, T)$ in the sense of traces.

We are now ready to state the main theorem of this paper.

Theorem 1.1. Let $\Omega \subset \mathbb{R}^{3}$ be a bounded domain with Lipschitz boundary. Let $\mathbf{v}_{0} \in$ $L_{0, \text { div }}^{2}(\Omega), \mathbf{T}_{0} \in L_{\text {sym }}^{2}(\Omega)$ and let $\varepsilon, \mu_{0}, T$ be given positive constants. Let $\mu: \mathbb{R}^{3 \times 3} \rightarrow \mathbb{R}^{+}$ be a continuous function satisfying (1.6)-(1.8) and let $\gamma: \mathbb{R}^{3 \times 3 \times 3} \rightarrow \mathbb{R}^{+}$be a continuous function satisfying (1.9)-(1.11). Moreover, let

$$
\begin{aligned}
\frac{6}{5}<p & \leq 2, & & \geq 4, \\
2 & <p, & q & >\frac{2 p}{p-1} .
\end{aligned}
$$

Then there exists a weak solution to system (1.3) with initial conditions (1.4) and boundary conditions (1.5). 
The rest of the paper is devoted to the proof of Theorem 1.1. The proof is organized as follows. In Section 2 we present the approximative system, show the existence of weak solutions to it and derive a-priori estimates. In Section 3 we perform the first limiting procedure, pass to the limit in most of the terms in the equations and identify the main problem which we have to solve. In Section 4 we recall some recently proved properties of the Lipschitz truncation operator which is a key tool in the final step of the proof. Then we complete the proof.

\section{Approximation AND A PRIORI ESTIMATES}

Let $\xi \in \mathcal{C}^{\infty}([0, \infty))$ be a non-increasing function such that $\xi \equiv 1$ in $[0,1], \xi \equiv 0$ in $[2, \infty)$ and $0 \geq \xi^{\prime} \geq-2$. For $m \in \mathbb{N}$ we define

$$
\xi^{m}(s):=\xi\left(\frac{s}{m}\right), \quad s \in[0, \infty) .
$$

We consider the approximative system (denoting $\left.\mathbf{D}^{m}=\mathbf{D}\left(\mathbf{v}^{m}\right), \mathbf{T}_{M}^{m}(t)=\frac{1}{|\Omega|} \int_{\Omega} \mathbf{T}^{m}(t, \cdot) \mathrm{d} \mathbf{x}\right)$

$$
\begin{gathered}
\operatorname{div} \mathbf{v}^{m}=0, \\
\partial_{t} \mathbf{v}^{m}+\operatorname{div}\left(\mathbf{v}^{m} \otimes \mathbf{v}^{m} \xi^{m}\left(\left|\mathbf{v}^{m}\right|\right)\right)+\nabla \pi^{m}-\operatorname{div}\left(\mu\left(\mathbf{D}^{m}\right) \mathbf{D}^{m}\right)=\operatorname{div} \mathbf{T}^{m}, \\
\partial_{t} \mathbf{T}^{m}+\mathbf{v}^{m} \cdot \nabla \mathbf{T}^{m}-\varepsilon \operatorname{div}\left(\gamma\left(\nabla \mathbf{T}^{m}\right) \nabla \mathbf{T}^{m}\right) \\
+\frac{1}{m}\left|\mathbf{T}_{M}^{m}\right|^{q-2} \mathbf{T}^{m}+\mathbf{T}^{m}=2 \mu_{0} \mathbf{D}^{m}+\mathbf{B}\left(\mathbf{v}^{m}, \mathbf{T}^{m}\right)
\end{gathered}
$$

with initial conditions (1.4) and boundary conditions (1.5).

Definition 2.1. By a weak solution to system (2.1) we mean a couple $\left(\mathbf{v}^{m}, \mathbf{T}^{m}\right)$ such that

$$
\begin{aligned}
\mathbf{v}^{m} & \in \mathcal{C}\left([0, T] ; L_{0, \operatorname{div}}^{2}(\Omega)\right) \cap L^{p}\left(0, T ; W_{0, \operatorname{div}}^{1, p}(\Omega)\right), \\
\mathbf{T}^{m} & \in \mathcal{C}\left([0, T] ; L_{\mathrm{sym}}^{2}(\Omega)\right) \cap L^{q}\left(0, T ; W_{\mathrm{sym}}^{1, q}(\Omega)\right), \\
\partial_{t} \mathbf{v}^{m} & \in L^{p^{\prime}}\left(0, T ;\left(W_{0, \operatorname{div}}^{1, p}(\Omega)\right)^{*}\right), \\
\partial_{t} \mathbf{T}^{m} & \in L^{q^{\prime}}\left(0, T ;\left(W_{\mathrm{sym}}^{1, q}(\Omega)\right)^{*}\right)
\end{aligned}
$$

satisfying

$$
\begin{array}{r}
\int_{0}^{T}\left\langle\partial_{t} \mathbf{v}^{m}, \boldsymbol{\varphi}\right\rangle_{1, p, \mathrm{div}} \mathrm{d} t-\int_{0}^{T} \int_{\Omega}\left(\mathbf{v}^{m} \otimes \mathbf{v}^{m} \xi^{m}\left(\left|\mathbf{v}^{m}\right|\right)\right): \nabla \boldsymbol{\varphi} \mathrm{d} \mathbf{x} \mathrm{d} t \\
\quad+\int_{0}^{T} \int_{\Omega} \mu\left(\mathbf{D}^{m}\right) \mathbf{D}^{m}: \nabla \boldsymbol{\varphi} \mathrm{d} \mathbf{x} \mathrm{d} t=\int_{0}^{T} \int_{\Omega} \operatorname{div} \mathbf{T}^{m} \cdot \boldsymbol{\varphi} \mathrm{d} \mathbf{x} \mathrm{d} t
\end{array}
$$


for all $\varphi \in L^{p}\left(0, T ; W_{0, \operatorname{div}}^{1, p}(\Omega)\right)$,

$$
\begin{array}{r}
\int_{0}^{T}\left\langle\partial_{t} \mathbf{T}^{m}, \boldsymbol{\psi}\right\rangle_{1, q} \mathrm{~d} t+\int_{0}^{T} \int_{\Omega}\left(\mathbf{v}^{m} \cdot \nabla \mathbf{T}^{m}\right): \boldsymbol{\psi} \mathrm{d} \mathbf{x} \mathrm{d} t \\
+\varepsilon \int_{0}^{T} \int_{\Omega} \gamma\left(\nabla \mathbf{T}^{m}\right) \nabla \mathbf{T}^{m}: \nabla \boldsymbol{\psi} \mathrm{d} \mathbf{x} \mathrm{d} t+\int_{0}^{T} \int_{\Omega}\left(\frac{1}{m}\left|\mathbf{T}_{M}^{m}\right|^{q-2} \mathbf{T}^{m}+\mathbf{T}^{m}\right): \boldsymbol{\psi} \mathrm{d} \mathbf{x} \mathrm{d} t \\
=2 \mu_{0} \int_{0}^{T} \int_{\Omega} \mathbf{D}^{m}: \boldsymbol{\psi} \mathrm{d} \mathbf{x} \mathrm{d} t-\int_{0}^{T} \int_{\Omega} \mathbf{v}^{m} \cdot \operatorname{div} \mathbf{A}\left(\mathbf{T}^{m}, \boldsymbol{\psi}\right) \mathrm{d} \mathbf{x} \mathrm{d} t
\end{array}
$$

for all $\boldsymbol{\psi} \in L^{q}\left(0, T ; W_{\mathrm{sym}}^{1, q}(\Omega)\right)$.

Theorem 2.1. Let $\Omega \subset \mathbb{R}^{3}$ be a bounded domain with Lipschitz boundary. Let $\mathbf{v}_{0} \in$ $L_{0, \text { div }}^{2}(\Omega), \mathbf{T}_{0} \in L_{\text {sym }}^{2}(\Omega)$ and let $\varepsilon, \mu_{0}$, $T$ be positive constants, $m \in \mathbb{N}$. Let $\mu: \mathbb{R}^{3 \times 3} \rightarrow \mathbb{R}^{+}$ be a continuous function satisfying (1.6)-(1.8) and let $\gamma: \mathbb{R}^{3 \times 3 \times 3} \rightarrow \mathbb{R}^{+}$be a continuous function satisfying (1.9)-(1.11). Moreover, let (1.18) hold. Then there exists a weak solution of system (2.1) with initial conditions (1.4) and boundary conditions (1.5).

Proof of Theorem 2.1. The existence is proved using the standard Galerkin method. We look for the approximations in the form

$$
\begin{aligned}
\mathbf{v}^{m, n}(t, \mathbf{x}) & =\sum_{i=1}^{n} c_{i}^{n}(t) \mathbf{w}^{i}(\mathbf{x}), \\
\mathbf{T}^{m, n}(t, \mathbf{x}) & =\sum_{i=1}^{n} d_{i}^{n}(t) \mathbf{W}^{i}(\mathbf{x}),
\end{aligned}
$$

where $\left\{\mathbf{w}^{i}(x)\right\}_{i=1}^{\infty}$ is an orthogonal system in $W_{0, \text { div }}^{1,2}(\Omega)$, orthonormal in $L^{2}(\Omega)$ formed by smooth functions and $\left\{\mathbf{W}^{i}(x)\right\}_{i=1}^{\infty}$ is an orthogonal system in $W_{\text {sym }}^{1,2}(\Omega)$, orthonormal in $L^{2}(\Omega)$ formed by smooth functions such that $\mathbf{W}^{1}(\mathbf{x})=\frac{1}{\sqrt{3|\Omega|}} \mathbf{1}_{\Omega}(\mathbf{x}) \mathbf{I}$, where $\mathbf{1}_{\Omega}(\mathbf{x})$ is the characteristic function of the domain $\Omega$ and $\mathbf{I}$ denotes the $3 \times 3$ identity matrix. The definition of the Galerkin approximation is standard. Local in time existence for solution to the Galerkin approximation is a direct consequence of the theory of the systems of ODEs. The solutions are global as soon as we are able to prove certain a priori estimates. If we "test" the Galerkin approximation for $\mathbf{v}^{m, n}$ by $2 \mu_{0} \mathbf{v}^{m, n}$ and the approximation for $\mathbf{T}^{m, n}$ by $\mathbf{T}^{m, n}$ and add the formulation for $\mathbf{T}^{m, n}$ "tested" by $\mathbf{W}^{1}$ (i.e. integrated over $\Omega$ ) and multiplied by $\mathbf{T}_{M}^{m, n}$, we get

$$
\begin{aligned}
& \frac{1}{2} \frac{\mathrm{d}}{\mathrm{dt}}\left(2 \mu_{0}\left\|\mathbf{v}^{m, n}\right\|_{2}^{2}+\left\|\mathbf{T}^{m, n}\right\|_{2}^{2}+\sqrt{\frac{|\Omega|}{3}}\left|\mathbf{T}_{M}^{m, n}\right|^{2}\right) \\
& +2 \mu_{0} c\left\|\nabla \mathbf{v}^{m, n}\right\|_{p}^{p}+\varepsilon c\left\|\nabla \mathbf{T}^{m, n}\right\|_{q}^{q}+\frac{c}{m}\left|\mathbf{T}_{M}^{m, n}\right|^{q} \\
& \leq C(t)+c \int_{\Omega}\left|\mathbf{v}^{m, n}\right|\left|\nabla \mathbf{T}^{m, n}\right|\left(\left|\mathbf{T}^{m, n}\right|+\left|\mathbf{T}_{M}^{m, n}\right|\right) \mathrm{d} \mathbf{x} .
\end{aligned}
$$

Under assumptions of the main theorem, it is an easy matter to estimate the integral on the right-hand side and we get control of norms coming from the left-hand side. The procedure is similar to estimates in Theorem 2.2, only slightly easier. Note, however, that the control depends on $m$; indeed, it is independent of $n$. Next, by duality argument, we also prove 
estimates of the time derivatives of $\mathbf{v}^{m, n}$ and $\mathbf{T}^{m, n}$ as stated in Definition 2.1 and using the Aubin-Lions lemma we get strong convergence of $\mathbf{v}^{m, n}$ and $\mathbf{T}^{m, n}$ in $L^{2}((0, T) \times \Omega)$. The proof of Theorem 2.1 is completed by the standard monotonicity argument (the Minty trick, see also Section 3) and the density argument to extend the class of the test functions.

Theorem 2.2. Under the assumptions of Theorem 2.1, for any $m \in \mathbb{N}$, we have the following estimates

$$
\left\|\mathbf{v}^{m}\right\|_{L^{\infty}\left(0, T ; L^{2}(\Omega)\right)}+\left\|\mathbf{T}^{m}\right\|_{L^{\infty}\left(0, T ; L^{2}(\Omega)\right)}+\left\|\nabla \mathbf{v}^{m}\right\|_{L^{p}\left(0, T ; L^{p}(\Omega)\right)}+\left\|\nabla \mathbf{T}^{m}\right\|_{L^{q}\left(0, T ; L^{q}(\Omega)\right)} \leq c
$$

with $c$ independent of $m$.

Proof of Theorem 2.2. For fixed $m$, we proceed similarly as above. However, we cannot use the information from the term $\frac{1}{m}\left|\mathbf{T}_{M}^{m}\right|^{q-2} \mathbf{T}^{m}$, hence the estimates are slightly more complex. Unlike the Galerkin approximation, we keep the estimates separately. We will not subtract two terms, however, they are of lower order and do not cause any troubles. We test equation $(2.1)_{2}$ by $\mathbf{v}^{m}$ and get

$$
\frac{1}{2} \int_{0}^{s} \frac{\mathrm{d}}{\mathrm{dt}}\left\|\mathbf{v}^{m}\right\|_{2}^{2} \mathrm{~d} t+c \int_{0}^{s}\left\|\nabla \mathbf{v}^{m}\right\|_{p}^{p} \mathrm{~d} t \leq \int_{0}^{s} \int_{\Omega} \varphi_{1} \mathrm{~d} \mathbf{x} \mathrm{d} t+\int_{0}^{s} \int_{\Omega}\left|\mathbf{v}^{m} \| \nabla \mathbf{T}^{m}\right| \mathrm{d} \mathbf{x} \mathrm{d} t .
$$

Next we test equation $(2.1)_{3}$ by $\mathbf{T}^{m}$ and have

$$
\begin{aligned}
& \frac{1}{2} \int_{0}^{s} \frac{\mathrm{d}}{\mathrm{dt}}\left\|\mathbf{T}^{m}\right\|_{2}^{2} \mathrm{~d} t+\varepsilon \int_{0}^{s}\left\|\nabla \mathbf{T}^{m}\right\|_{q}^{q} \mathrm{~d} t+\int_{0}^{s}\left\|\mathbf{T}^{m}\right\|_{2}^{2} \mathrm{~d} t \\
& \leq \int_{0}^{s} \int_{\Omega} \varphi_{3} \mathrm{~d} \mathbf{x} \mathrm{d} t+\int_{0}^{s} \int_{\Omega}\left|\mathbf{v}^{m}\right|\left|\nabla \mathbf{T}^{m}\right| \mathrm{d} \mathbf{x} \mathrm{d} t+\int_{0}^{s} \int_{\Omega}\left|\mathbf{v}^{m}\right|\left|\mathbf{T}^{m} \| \nabla \mathbf{T}^{m}\right| \mathrm{d} \mathbf{x} \mathrm{d} t .
\end{aligned}
$$

The information about $\mathbf{T}^{m}$ on the left-hand side is not sufficient, as it is in the second power. We therefore use the same trick as before; we integrate (2.1) 3 over $\Omega$ and multiply it by $\mathbf{T}_{M}^{m}$. Hence

$$
\frac{1}{2} \int_{0}^{s} \frac{\mathrm{d}}{\mathrm{dt}}\left|\mathbf{T}_{M}^{m}\right|^{2} \mathrm{~d} t+\int_{0}^{s}\left|\mathbf{T}_{M}^{m}\right|^{2} \mathrm{~d} t \leq c \int_{0}^{s}\left|\mathbf{T}_{M}^{m}\right| \int_{\Omega}\left|\mathbf{v}^{m}\right|\left|\nabla \mathbf{T}^{m}\right| \mathrm{d} \mathbf{x} \mathrm{d} t .
$$

We first take $p \leq 2$. Then we have from (2.8)

$$
\left\|\mathbf{T}_{M}^{m}\right\|_{L^{\infty}(0, s)} \leq C\left(1+\int_{0}^{s}\left\|\mathbf{v}^{m}\right\|_{L^{2}(\Omega)}\left\|\nabla \mathbf{T}^{m}\right\|_{L^{q}(\Omega)} \mathrm{d} t\right) .
$$

Hence, writing the last term in (2.7) as

$$
\begin{aligned}
\int_{0}^{s} \int_{\Omega}\left|\mathbf{v}^{m}\right|\left|\mathbf{T}^{m}\right| \mid & \nabla \mathbf{T}^{m} \mid \mathrm{d} \mathbf{x} \mathrm{d} t \\
& \leq \int_{0}^{s} \int_{\Omega}\left|\mathbf{v}^{m}\right|\left|\mathbf{T}^{m}-\mathbf{T}_{M}^{m}\right|\left|\nabla \mathbf{T}^{m}\right| \mathrm{d} \mathbf{x} \mathrm{d} t+\int_{0}^{s}\left|\mathbf{T}_{M}^{m}\right| \int_{\Omega}\left|\mathbf{v}^{m}\right|\left|\nabla \mathbf{T}^{m}\right| \mathrm{d} \mathbf{x} \mathrm{d} t
\end{aligned}
$$


we have using Poincaré's inequality

$$
\begin{aligned}
\left\|\nabla \mathbf{T}^{m}\right\|_{L^{q}\left(0, s ; L^{q}(\Omega)\right)}^{q} \leq & C\left(1+\left(\int_{0}^{s}\left\|\mathbf{v}^{m}\right\|_{L^{2}(\Omega)}^{2} \mathrm{~d} t\right)^{\frac{1}{2}}\left(\int_{0}^{s}\left\|\nabla \mathbf{T}^{m}\right\|_{L^{q}(\Omega)}^{q} \mathrm{~d} t\right)^{\frac{1}{q}}\right. \\
& +\left(\int_{0}^{s}\left\|\mathbf{v}^{m}\right\|_{L^{2}(\Omega)}^{2} \mathrm{~d} t\right)^{\frac{1}{2}}\left(\int_{0}^{s}\left\|\nabla \mathbf{T}^{m}\right\|_{L^{q}(\Omega)}^{q} \mathrm{~d} t\right)^{\frac{2}{q}} \\
& \left.+\left(\int_{0}^{s}\left\|\mathbf{v}^{m}\right\|_{L^{2}(\Omega)}^{2} \mathrm{~d} t\right)\left(\int_{0}^{s}\left\|\nabla \mathbf{T}^{m}\right\|_{L^{q}(\Omega)}^{q} \mathrm{~d} t\right)^{\frac{2}{q}}\right) .
\end{aligned}
$$

Therefore

$$
\left\|\nabla \mathbf{T}^{m}\right\|_{L^{q}\left(0, T ; L^{q}(\Omega)\right)} \leq C\left(1+\left(\int_{0}^{s}\left\|\mathbf{v}^{m}\right\|_{L^{2}(\Omega)}^{2} \mathrm{~d} t\right)^{\frac{1}{q-2}}\right) .
$$

Finally, from (2.6)

$$
\left\|\mathbf{v}^{m}(s, \cdot)\right\|_{L^{2}(\Omega)}^{2} \leq C\left(1+\left(\int_{0}^{s}\left\|\mathbf{v}^{m}\right\|_{L^{2}(\Omega)}^{2} \mathrm{~d} t\right)^{\frac{1}{2}+\frac{1}{q-2}}\right)
$$

which leads to the estimate by virtue of the Gronwall lemma provided $q \geq 4$. For $p>2$ we use the $L^{p}$-norm of the velocity gradient. Proceeding similarly as above we get

$$
\left\|\nabla \mathbf{v}^{m}\right\|_{L^{p}\left(0, T ; L^{p}(\Omega)\right)}^{p} \leq C\left(1+\left(\left\|\nabla \mathbf{v}^{m}\right\|_{L^{p}\left(0, T ; L^{p}(\Omega)\right)}\right)^{1+\frac{2}{q-2}}\right)
$$

which gives the required a-priori estimates provided $q>\frac{2 p}{p-1}$.

\section{Limiting PROCEDURE I}

In what follows, we consider only the more interesting case $p \leq 2$. The other case can be proved similarly, we only need to work with different spaces corresponding to the a-priori estimates.

Lemma 3.1. Let $\left\{\left(\mathbf{v}^{m}, \mathbf{T}^{m}\right)\right\}_{m=1}^{\infty}$ be a sequence of weak solutions of (2.1). Then there exists a subsequence (not relabeled) such that

$$
\begin{aligned}
& \mathbf{v}^{m} \rightarrow \mathbf{v} \text { weakly in } L^{p}\left(0, T ; W_{0, \operatorname{div}}^{1, p}(\Omega)\right), \\
& \mathbf{v}^{m} \rightarrow^{*} \mathbf{v} \text { weakly* in } L^{\infty}\left(0, T ; L^{2}(\Omega)\right) \text {, } \\
& \mathbf{T}^{m} \rightarrow \mathbf{T} \text { weakly in } L^{q}\left(0, T ; W_{\mathrm{sym}}^{1, q}(\Omega)\right), \\
& \mathbf{T}^{m} \rightarrow^{*} \mathbf{T} \text { weakly in } L^{\infty}\left(0, T ; L^{2}(\Omega)\right), \\
& \mu\left(\mathbf{D}^{m}\right) \mathbf{D}^{m} \rightarrow \mathbf{S} \text { weakly in } L^{p^{\prime}}\left(0, T ; L^{p^{\prime}}(\Omega)\right), \\
& \gamma\left(\nabla \mathbf{T}^{m}\right) \nabla \mathbf{T}^{m} \rightarrow \mathbf{U} \quad \text { weakly in } L^{q^{\prime}}\left(0, T ; L^{q^{\prime}}(\Omega)\right), \\
& \mathbf{v}^{m} \rightarrow \mathbf{v} \quad \text { weakly in } L^{\frac{5}{3} p}\left(0, T ; L^{\frac{5}{3} p}(\Omega)\right)
\end{aligned}
$$




$$
\begin{aligned}
\partial_{t} \mathbf{v}^{m} & \rightarrow \partial_{t} \mathbf{v} \quad \text { weakly in } L^{\sigma}\left(0, T ;\left(W_{0, \text { div }}^{1, \sigma^{\prime}}(\Omega)\right)^{*}\right), \quad 1 \leq \sigma \leq \frac{5}{6} p, \\
\partial_{t} \mathbf{T}^{m} & \rightarrow \partial_{t} \mathbf{T} \quad \text { weakly in } L^{q^{\prime}}\left(0, T ;\left(W_{\mathrm{sym}}^{1, q}(\Omega)\right)^{*}\right), \\
\mathbf{v}^{m} & \rightarrow \mathbf{v} \quad \text { strongly in } L^{p}\left(0, T ; L^{r}(\Omega)\right), \quad 1 \leq r<\frac{3 p}{3-p}, \\
\mathbf{T}^{m} & \rightarrow \mathbf{T} \text { strongly in } L^{q}\left(0, T ; L^{\bar{r}}(\Omega)\right), \quad 1 \leq \bar{r} \leq \infty \\
\mathbf{v}^{m} & \rightarrow \mathbf{v} \quad \text { strongly in } L^{2 \sigma}\left(0, T ; L^{2 \sigma}(\Omega)\right), \quad 1 \leq 2 \sigma<\frac{5}{3} p, \\
\mathbf{v}^{m} \otimes \mathbf{v}^{m} \xi^{m}\left(\left|\mathbf{v}^{m}\right|\right) & \rightarrow \mathbf{v} \otimes \mathbf{v} \text { strongly in } L^{\sigma}\left(0, T ; L^{\sigma}(\Omega)\right), \quad 1 \leq \sigma<\frac{5}{6} p, \\
\frac{1}{m}\left|\mathbf{T}_{M}^{m}\right|^{q-2} \mathbf{T}^{m} & \rightarrow \mathbf{0} \text { strongly in } L^{1}\left(0, T ; L^{1}(\Omega)\right) .
\end{aligned}
$$

Proof of Lemma 3.1. Convergences (3.1)-(3.4) are direct consequences of a-priori estimates (2.5). Convergences (3.5) and (3.6) are achieved combining a-priori estimate (2.5) with (1.7) and (1.10) respectively. Interpolating between $L^{\infty}\left(0, T ; L^{2}(\Omega)\right)$ and $L^{p}\left(0, T ; W_{0, \operatorname{div}}^{1, p}(\Omega)\right)$ yields (3.7).

Next we want to prove (3.8). To this aim it is enough to prove a priori bound for the time derivative of $\mathbf{v}^{m}$ in $L^{\sigma}\left(0, T ;\left(W_{0, \text { div }}^{1, \sigma^{\prime}}\right)^{*}\right)$. We denote

$$
\mathcal{B}_{1}:=\left\{\boldsymbol{\varphi} \in L^{\sigma^{\prime}}\left(0, T ; W_{0, \text { div }}^{1, \sigma^{\prime}}\right),\|\varphi\|_{L^{\sigma^{\prime}}\left(0, T ; W_{0, \text { div }}^{1, \sigma^{\prime}}\right)} \leq 1\right\}
$$

and using (2.2) we estimate

$$
\begin{aligned}
& \left\|\partial_{t} \mathbf{v}^{m}\right\|_{L^{\sigma}\left(0, T ;\left(W_{0, \text { div }}^{1, \sigma^{\prime}}\right)^{*}\right)}=\sup _{\varphi \in \mathcal{B}_{1}}\left|\int_{0}^{T}\left\langle\partial_{t} \mathbf{v}^{m}, \boldsymbol{\varphi}\right\rangle_{1, \sigma^{\prime}, \operatorname{div}} \mathrm{d} t\right| \\
& =\sup _{\psi \in \mathcal{B}_{1}}\left|\int_{Q_{T}}\left(\mathbf{v}^{m} \otimes \mathbf{v}^{m} \xi^{m}\left(\left|\mathbf{v}^{m}\right|\right)-\mu\left(\mathbf{D}^{m}\right) \mathbf{D}^{m}-\mathbf{T}^{m}\right): \nabla \boldsymbol{\varphi} \mathrm{d} \mathbf{x} \mathrm{d} t\right| \\
& \leq \sup _{\boldsymbol{\psi} \in \mathcal{B}_{1}}\left(\left\|\mathbf{v}^{m}\right\|_{L^{2 \sigma}\left(Q_{T}\right)}^{2}+c\left\|\mathbf{D}^{m}\right\|_{L^{(p-1) \sigma}\left(Q_{T}\right)}^{p-1}+\left\|\varphi_{2}\right\|_{L^{\sigma}\left(Q_{T}\right)}+\left\|\mathbf{T}^{m}\right\|_{L^{\sigma}\left(Q_{T}\right)}\right)\|\nabla \boldsymbol{\varphi}\|_{L^{\sigma^{\prime}}\left(Q_{T}\right)} \\
& \leq c\left(1+\left\|\mathbf{v}^{m}\right\|_{L^{\frac{5}{3} p}\left(0, T ; L^{\frac{5}{3} p}(\Omega)\right)}^{2}+\left\|\mathbf{D}^{m}\right\|_{L^{(p-1) \sigma}\left(0, T ; L^{(p-1) \sigma}(\Omega)\right)}^{p-1}+\left\|\mathbf{T}^{m}\right\|_{L^{2}\left(0, T ; L^{2}(\Omega)\right)}\right) \\
& \leq c\left(1+\left\|\mathbf{v}^{m}\right\|_{L^{\infty}\left(0, T ; L^{2}(\Omega)\right)}^{2}+\left\|\nabla \mathbf{v}^{m}\right\|_{L^{p}\left(0, T ; L^{p}(\Omega)\right)}^{2}+\left\|\mathbf{D}^{m}\right\|_{L^{p}\left(0, T ; L^{p}(\Omega)\right)}^{p-1}+\left\|\mathbf{T}^{m}\right\|_{L^{q}\left(0, T ; L^{q}(\Omega)\right)}\right) \\
& \leq c
\end{aligned}
$$

we used $\sigma \leq \frac{5}{6} p<2$ when we estimated $\left\|\varphi_{2}\right\|$ and $\left\|\mathbf{T}^{m}\right\|$ in $L^{\sigma}\left(0, T ; L^{\sigma}(\Omega)\right)$ and $\left\|\mathbf{D}^{m}\right\|$ in $L^{(p-1) \sigma}\left(0, T ; L^{(p-1) \sigma}(\Omega)\right)$. Convergence (3.8) now follows easily. In the same way we derive also (3.9) using (2.3). Having estimates for time derivatives, convergences (3.10) and (3.11) are direct consequences of Aubin-Lions lemma. Convergence (3.12) follows from (3.7) and 
(3.10) with $r=p$, by interpolation. Further, (3.12) implies that $\mathbf{v}^{m}(\mathbf{x}, t) \rightarrow \mathbf{v}(\mathbf{x}, t)$ a.e. in $Q_{T}$, which together with the uniform estimate

$$
\int_{Q_{T}}\left|\mathbf{v}^{m} \otimes \mathbf{v}^{m} \xi^{m}\left(\left|\mathbf{v}^{m}\right|\right)\right|^{\sigma} \mathrm{d} \mathbf{x} \mathrm{d} t \leq \int_{Q_{T}}\left|\mathbf{v}^{m}\right|^{2 \sigma} \mathrm{d} \mathbf{x} \mathrm{d} t \leq c
$$

and a combination of Lebesgue's and Vitali's theorem imply the strong convergence (3.13). Finally, (3.14) is a direct consequence of the bound $\frac{1}{m}\left\|\mathbf{T}_{M}^{m}\right\|_{L^{\infty}(0, T)}^{q-1} \leq C$ and the estimates above.

Now we pass to the limit in equation (2.3) for the extra stress tensor T. For this reason fix a test function $\psi \in L^{q}\left(0, T ; W_{\text {sym }}^{1, q}(\Omega)\right)$. Using Lemma 3.1 we claim that we can pass to the limit in all terms in equation (2.3). For example in the convective term

$$
\int_{Q_{T}} \mathbf{v}^{m} \cdot \nabla \mathbf{T}^{m}: \boldsymbol{\psi} \mathrm{d} \mathbf{x} \mathrm{d} t
$$

we use strong convergence (3.12) and weak convergence $\nabla \mathbf{T}^{m} \rightarrow \nabla \mathbf{T}$ in $L^{q}\left(Q_{T}\right)$ keeping in mind that $\frac{1}{2 \sigma}+\frac{2}{q} \leq 1$ as $q \geq 4$. Exactly the same argument applies also for the terms

$$
\int_{Q_{T}} \mathbf{v}^{m} \cdot \operatorname{div} \mathbf{A}\left(\mathbf{T}^{m}, \boldsymbol{\psi}\right) \mathrm{d} \mathbf{x} \mathrm{d} t
$$

and thus the limit equation is

$$
\begin{array}{r}
\int_{0}^{T}\left\langle\partial_{t} \mathbf{T}, \boldsymbol{\psi}\right\rangle_{1, q} \mathrm{~d} t+\int_{0}^{T} \int_{\Omega}(\mathbf{v} \cdot \nabla \mathbf{T}): \boldsymbol{\psi} \mathrm{d} \mathbf{x} \mathrm{d} t \\
+\varepsilon \int_{0}^{T} \int_{\Omega} \mathbf{U}: \nabla \boldsymbol{\psi} \mathrm{d} \mathbf{x} \mathrm{d} t+\int_{0}^{T} \int_{\Omega} \mathbf{T}: \boldsymbol{\psi} \mathrm{d} \mathbf{x} \mathrm{d} t \\
=2 \mu_{0} \int_{0}^{T} \int_{\Omega} \mathbf{D}: \boldsymbol{\psi} \mathrm{d} \mathbf{x} \mathrm{d} t-\int_{0}^{T} \int_{\Omega} \mathbf{v} \cdot \operatorname{div} \mathbf{A}(\mathbf{T}, \boldsymbol{\psi}) \mathrm{d} \mathbf{x} \mathrm{d} t
\end{array}
$$

for all $\boldsymbol{\psi} \in L^{q}\left(0, T ; W_{\mathrm{sym}}^{1, q}(\Omega)\right)$. Finally we have to show that

$$
\mathbf{U}=\gamma(\nabla \mathbf{T}) \nabla \mathbf{T}
$$

To this end we use $\mathbf{T}^{m}$ as a test function in (2.3) and $\mathbf{T}$ as a test function in (3.15). Note that these are both suitable test functions in the equations. Comparing the results we get

$$
\limsup _{m \rightarrow \infty} \int_{Q_{T}} \gamma\left(\nabla \mathbf{T}^{m}\right) \nabla \mathbf{T}^{m}: \nabla \mathbf{T}^{m} \mathrm{~d} \mathbf{x} \mathrm{d} t=\int_{Q_{T}} \mathbf{U}: \nabla \mathbf{T} \mathrm{d} \mathbf{x} \mathrm{d} t .
$$

Using the monotonicity assumption for $\gamma$ (1.11) we get

$$
0 \leq \int_{Q_{T}}\left(\gamma\left(\nabla \mathbf{T}^{m}\right) \nabla \mathbf{T}^{m}-\gamma(\nabla \mathbf{C}) \nabla \mathbf{C}\right):\left(\nabla \mathbf{T}^{m}-\nabla \mathbf{C}\right) \mathrm{d} \mathbf{x} \mathrm{d} t
$$

and taking the limsup also

$$
0 \leq \int_{Q_{T}}(\mathbf{U}-\gamma(\nabla \mathbf{C}) \nabla \mathbf{C}):(\nabla \mathbf{T}-\nabla \mathbf{C}) \mathrm{d} \mathbf{x} \mathrm{d} t
$$


which holds for all $\mathbf{C} \in L^{q}\left(0, T ; W_{\text {sym }}^{1, q}(\Omega)\right)$. Thus we can plug in $\mathbf{C}=\mathbf{T}+\beta \mathbf{E}$ for $\beta \in \mathbb{R}$ and any $\mathbf{E} \in L^{q}\left(0, T ; W_{\text {sym }}^{1, q}(\Omega)\right)$. Then letting $\beta \rightarrow 0_{+}$and $\beta \rightarrow 0_{-}$and using the continuity of the function $\gamma$ we finally arrive at

$$
0=\int_{Q_{T}}(\mathbf{U}-\gamma(\nabla \mathbf{T}) \nabla \mathbf{T}): \mathbf{E} \mathrm{d} \mathbf{x} \mathrm{d} t
$$

which yields (3.16).

Next we pass to the limit in the momentum equation (2.2). Here we fix a test function $\boldsymbol{\varphi} \in L^{\sigma^{\prime}}\left(0, T ; W_{0, \text { div }}^{1, \sigma^{\prime}}(\Omega)\right)$ with $\sigma^{\prime}$ being a dual exponent to $\sigma \in\left(1, \frac{5 p}{6}\right]$. Using convergences stated in Lemma 3.1 we can pass to the limit in all terms of (2.2) and arrive at

$$
\begin{array}{r}
\int_{0}^{T}\left\langle\partial_{t} \mathbf{v}, \boldsymbol{\varphi}\right\rangle_{1, p, \mathrm{div}} \mathrm{d} t-\int_{0}^{T} \int_{\Omega}(\mathbf{v} \otimes \mathbf{v}): \nabla \boldsymbol{\varphi} \mathrm{d} \mathbf{x} \mathrm{d} t \\
\quad+\int_{0}^{T} \int_{\Omega} \mathbf{S}: \nabla \boldsymbol{\varphi} \mathrm{d} \mathbf{x} \mathrm{d} t=\int_{0}^{T} \int_{\Omega} \operatorname{div} \mathbf{T} \cdot \boldsymbol{\varphi} \mathrm{d} \mathbf{x} \mathrm{d} t
\end{array}
$$

for all $\boldsymbol{\varphi} \in L^{\sigma^{\prime}}\left(0, T ; W_{0, \text { div }}^{1, \sigma^{\prime}}(\Omega)\right)$. It remains to show that

$$
\mathbf{S}=\mu(\mathbf{D}) \mathbf{D}
$$

\section{Lipschitz TRUNCATION AND Limiting PROCEDURE II}

To prove (3.18) is not as easy as showing (3.16). Recall that in this case $\mathbf{v}$ is not a suitable test function in the limit equation (3.17). The rest of the paper is thus devoted to the proof (3.18).

In order to show the convergence

$$
\mu\left(\mathbf{D}^{m}\right) \mathbf{D}^{m} \rightarrow \mu(\mathbf{D}) \mathbf{D} \quad \text { a.e. in } Q_{T}
$$

(i.e. $\mathbf{S}=\mu(\mathbf{D}) \mathbf{D})$, we will use Theorem 2.16 and Corollary 2.17 from [1]. We first introduce certain notation. For $\alpha>0$ we say that $Q=I \times B \subset \mathbb{R} \times \mathbb{R}^{3}$ is an $\alpha$-parabolic cylinder, if $r_{I}=\alpha r_{B}^{2}$, where $r_{I}$ is the radius of the interval $I$ and $r_{B}$ the radius of the ball $B$. By $\mathcal{Q}^{\alpha}$ we denote the set of all $\alpha$-parabolic cylinders. For $\kappa>0$ we denote $\kappa Q$ the scaled cylinder $\kappa Q=(\kappa I) \times(\kappa B)$, where $\kappa B$ is the scaled ball with the same center, similarly $\kappa I$. Then $\alpha$-parabolic maximal operators $\mathcal{M}^{\alpha}$ and $\mathcal{M}_{s}^{\alpha}, s \in[1, \infty)$ are defined

$$
\begin{gathered}
\left(\mathcal{M}^{\alpha} f\right)(t, \mathbf{x}):=\sup _{Q^{\prime} \in \mathcal{Q}^{\alpha} ;(t, \mathbf{x}) \in Q^{\prime}} \frac{1}{\left|Q^{\prime}\right|} \int_{Q^{\prime}}|f(s, \mathbf{y})| \mathrm{d} s \mathrm{~d} \mathbf{y} \\
\left(\mathcal{M}_{s}^{\alpha} f\right)(t, \mathbf{x}):=\left(\left(\mathcal{M}^{\alpha}|f|^{s}\right)(t, \mathbf{x})\right)^{\frac{1}{s}} .
\end{gathered}
$$

For $\lambda, \alpha>0$ and $\sigma>1$ we define

$$
\mathcal{O}_{\lambda}^{\alpha}(\mathbf{z}):=\left\{(t, \mathbf{x}) ;\left(\mathcal{M}_{\sigma}^{\alpha}\left(\xi_{\frac{1}{3} Q_{0}}\left|\nabla^{2} \mathbf{z}\right|\right)>\lambda \cap\left(\mathcal{M}_{\sigma}^{\alpha}\left(\xi_{\frac{1}{3} Q_{0}}\left|\partial_{t} \mathbf{z}\right|\right)>\lambda\right\} .\right.\right.
$$

Note that $\mathbf{z} \sim \nabla^{-1} \mathbf{u}$; for more precise definition of $\mathbf{z}$ see the proof of Theorem 2.16 in [1].

We have (see Theorem 2.16 and Corollary 2.17 in [1]) 
Theorem 4.1. Let $1<p<\infty, p, p^{\prime}>\sigma$. Let $\mathbf{u}_{m}$ and $\mathbf{G}_{m}$ satisfy

$$
\left\langle\partial_{t} \mathbf{u}_{m}, \boldsymbol{\varphi}\right\rangle=\left\langle\operatorname{div} \mathbf{G}_{m}, \boldsymbol{\varphi}\right\rangle
$$

for all $\boldsymbol{\varphi} \in C_{0 \text {,div }}^{\infty}\left(Q_{0}\right), Q_{0}=I_{0} \times B_{0} \subset \mathbb{R} \times \mathbb{R}^{3}$. Assume that $\mathbf{u}_{m}$ is a weak null sequence in $L^{p}\left(I_{0} ; W^{1, p}\left(B_{0}\right)\right)$ and a strong null sequence in $L^{\sigma}\left(Q_{0}\right)$ and bounded in $L^{\infty}\left(I_{0} ; L^{\sigma}\left(B_{0}\right)\right)$. Further assume that $\mathbf{G}_{m}=\mathbf{G}_{1, m}+\mathbf{G}_{2, m}$ such that $\mathbf{G}_{1, m}$ is a weak null sequence in $L^{p^{\prime}}\left(Q_{0}\right)$ and $\mathbf{G}_{2, m}$ converges strongly to zero in $L^{\sigma}\left(Q_{0}\right)$. Then there exists a double sequence $\left\{\lambda_{m, k}\right\} \subset \mathbb{R}^{+}$and $k_{0} \in \mathbb{N}$ with

(a) $2^{2^{k}} \leq \lambda_{m, k} \leq 2^{2^{k+1}}$

such that the double sequence $\mathbf{u}_{m, k}:=\mathbf{u}_{\lambda_{m, k}}^{\alpha_{m, k}} \in L^{1}\left(Q_{0}\right), \alpha_{m, k}:=\lambda_{m, k}^{2-p}$ and $\mathcal{O}_{m, k}:=$ $\mathcal{O}_{\lambda_{m, k}}^{\alpha_{m, k}}$ defined above satisfy for all $k \geq k_{0}$

(b) $\mathbf{u}_{m, k} \in L^{s}\left(\frac{1}{4} I_{0} ; W_{0, \text { div }}^{1, s}\left(\frac{1}{6} B_{0}\right)\right)$ for all $s<\infty$ and supp $\mathbf{u}_{m, k} \subset \frac{1}{6} Q_{0}$

(c) $\mathbf{u}_{m, k}=\mathbf{u}_{m}$ a.e. on $\frac{1}{8} Q_{0} \backslash \mathcal{O}_{m, k}$

(d) $\left\|\nabla \mathbf{u}_{m, k}\right\|_{L^{\infty}\left(\frac{1}{4}\left(Q_{0}\right)\right)} \leq c \lambda_{m, k}$

(e) $\mathbf{u}_{m, k} \rightarrow 0$ in $L^{\infty}\left(\frac{1}{4} Q_{0}\right)$ for $m \rightarrow \infty$ and $k$ fixed

(f) $\nabla \mathbf{u}_{m, k} \rightarrow^{*} 0$ in $L^{\infty}\left(\frac{1}{4} Q_{0}\right)$ for $m \rightarrow \infty$ and $k$ fixed

(g) $\lim \sup _{m \rightarrow \infty} \lambda_{m, k}^{p}\left|\mathcal{O}_{m, k}\right| \leq c 2^{-k}$

(h) $\lim \sup _{m \rightarrow \infty}\left|\int_{Q_{0}} \mathbf{G}_{m}: \nabla \mathbf{u}_{m, k} \mathrm{~d} \mathbf{x} \mathrm{d} t\right| \leq c \lambda_{m, k}^{p}\left|\mathcal{O}_{m, k}\right|$

(i) Additionally, let $\zeta \in C_{0}^{\infty}\left(\frac{1}{6} Q_{0}\right)$ with $\chi_{\frac{1}{8} Q_{0}} \leq \zeta \leq \chi_{\frac{1}{6} Q_{0}}$. Let $\mathbf{u}_{m}$ be uniformly bounded in $L^{\infty}\left(I_{0} ; L^{\sigma}\left(B_{0}\right)\right)$, then for every $\mathbf{K} \in L^{p^{\prime}}\left(\frac{1}{6} Q_{0}\right)$

$$
\limsup _{m \rightarrow \infty}\left|\left(\int_{Q_{0}}\left(\mathbf{G}_{1, m}+\mathbf{K}\right): \nabla \mathbf{u}_{m}\right) \zeta \chi_{\mathcal{O}_{m, k}^{C}} \mathrm{~d} \mathbf{x} \mathrm{d} t\right| \leq c 2^{-\frac{k}{p}}
$$

We apply this theorem to our problem (2.1); cf. Theorem 3.1 in [1]. We denote $\mathbf{u}_{m}=$ $\mathbf{v}_{m}-\mathbf{v}$. Then

$$
\begin{gathered}
\mathbf{u}_{m} \rightarrow \mathbf{0} \quad \text { in } L^{p}\left(0, T ; W_{0, \text { div }}^{1, p}(\Omega)\right), \\
\mathbf{u}_{m} \rightarrow \mathbf{0} \quad \text { in } L^{2 \sigma}\left(Q_{T}\right), \\
\mathbf{u}_{m} \rightarrow^{*} \mathbf{0} \quad \text { in } L^{\infty}\left(0, T ; L^{2}(\Omega)\right),
\end{gathered}
$$

see (3.1), (3.12) and (3.2). Further

$$
\int_{0}^{T} \int_{\Omega} \mathbf{u}_{m} \cdot \partial_{t} \boldsymbol{\varphi} \mathrm{d} \mathbf{x} \mathrm{d} t=\int_{0}^{T} \int_{\Omega} \mathbf{G}_{m}: \nabla \boldsymbol{\varphi} \mathrm{d} \mathbf{x} \mathrm{d} t
$$

for all $\boldsymbol{\varphi} \in C_{0, \infty}^{\infty}\left(Q_{T}\right)$, where $\mathbf{G}_{m}=\mathbf{G}_{1, m}+\mathbf{G}_{2, m}$ with

$$
\begin{gathered}
\mathbf{G}_{1, m}=\mu\left(\mathbf{D}_{m}\right) \mathbf{D}_{m}-\mathbf{S}, \\
\mathbf{G}_{2, m}=-\mathbf{v}_{m} \otimes \mathbf{v}_{m} \xi\left(\left|\mathbf{v}_{m}\right|\right)+\mathbf{v} \otimes \mathbf{v}+\mathbf{T}^{m}-\mathbf{T} .
\end{gathered}
$$

We have $\left\|\mathbf{G}_{1, m}\right\|_{L^{p^{\prime}}\left(0, T ; L^{p^{\prime}}(\Omega)\right)} \leq C$ and $\mathbf{G}_{2, m} \rightarrow \mathbf{0}$ in $L^{\sigma_{1}}\left(Q_{T}\right)$ with $\sigma_{1}=\min \{2 \sigma, \bar{r}\}$, see (2.5), (3.11) and (3.13). 
Take now $Q \subset \subset(0, T) \times \Omega$. Due to properties mentioned above, assumptions of Theorem 4.1 (i) are fulfilled. Hence, plugging in $\mathbf{K}=\mathbf{S}-\mu(\mathbf{D}) \mathbf{D}$ we have for $\zeta \in C_{0}^{\infty}\left(\frac{1}{6} Q_{0}\right)$

$$
\limsup _{m \rightarrow \infty}\left|\int_{0}^{T} \int_{\Omega}\left(\mathbf{G}_{1, m}+\mathbf{S}-\mu(\mathbf{D}) \mathbf{D}\right): \nabla \mathbf{u}_{m} \zeta \chi_{\mathcal{O}_{m, k}^{C}} \mathrm{~d} \mathbf{x} \mathrm{d} t\right| \leq C 2^{-\frac{k}{p}} .
$$

Therefore

$$
\limsup _{m \rightarrow \infty}\left|\int_{0}^{T} \int_{\Omega}\left(\mu\left(\mathbf{D}_{m}\right) \mathbf{D}_{m}-\mu(\mathbf{D}) \mathbf{D}\right): \nabla \mathbf{u}_{m} \zeta \chi_{\mathcal{O}_{m, k}^{C}} \mathrm{~d} \mathbf{x} \mathrm{d} t\right| \leq C 2^{-\frac{k}{p}} .
$$

Take $\theta \in(0,1)$. By virtue of Hölder's inequality and Theorem 4.1 (g)

$$
\begin{aligned}
& \limsup _{m \rightarrow \infty}\left|\int_{0}^{T} \int_{\Omega}\left(\left(\mu\left(\mathbf{D}_{m}\right) \mathbf{D}_{m}-\mu(\mathbf{D}) \mathbf{D}\right): \nabla \mathbf{u}_{m}\right)^{\theta} \zeta \chi_{\mathcal{O}_{m, k}} \mathrm{~d} \mathbf{x} \mathrm{d} t\right| \\
& \quad \leq C \limsup _{m \rightarrow \infty}\left|\mathcal{O}_{k, m}\right|^{1-\theta} \leq C 2^{-(1-\theta) \frac{k}{p}} .
\end{aligned}
$$

Thus, both estimates imply

$$
\limsup _{m \rightarrow \infty}\left|\int_{0}^{T} \int_{\Omega}\left(\left(\mu\left(\mathbf{D}_{m}\right) \mathbf{D}_{m}-\mu(\mathbf{D}) \mathbf{D}\right): \nabla \mathbf{u}_{m}\right)^{\theta} \zeta \mathrm{d} \mathbf{x} \mathrm{d} t\right| \leq C 2^{-(1-\theta) \frac{k}{p}} .
$$

Taking $\lim _{k \rightarrow \infty}$ the right-hand side tends to zero. Using now standard approach from [3] (see also [6] or with more details [7]), due to the strict monotonicity of $\mu$, see (1.8), we get $\mu\left(\mathbf{D}_{m}\right) \mathbf{D}_{m} \rightarrow \mu(\mathbf{D}) \mathbf{D}$ a.e. in $\frac{1}{8} Q_{T}$ which implies that $\mathbf{S}=\mu(\mathbf{D}) \mathbf{D}$. The proof of the main theorem is finished.

\section{REFERENCES}

[1] Breit, D., Diening, L., Schwarzacher, S.: Solenoidal Lipschitz truncation for parabolic PDE's, arXiv: 1209.6522 v2 (2013).

[2] Constantin, P., Kliegl, M.: Note on global regularity for two-dimensional Oldroyd-B fluids with diffusive stress, Arch. Ration. Mech. Anal. 206 (3) (2012), 725-740.

[3] DalMaso, G., Murat, F.: Almost everywhere convergence of gradients of solutions to nonlinear elliptic systems. Nonlinear Anal. TMA 31 (3-4) (1998), 405-412.

[4] Diening, L., Růžička, M., Wolf, J.: Existence of Weak Solutions for Unsteady Motions of Generalized Newtonian Fluids, Ann. Sc. Norm. Super. Pisa, Cl. Sci. (5) 9 (2010), 1-46.

[5] Fang, D., Hieber, M. Zi, R.: Global existence results for Oldroyd-B fluids in exterior domains: the case of non-small coupling parameters, Math. Ann. 357 (2013), no. 2, 687-709.

[6] Frehse, J., Málek, J., Steinhauer, M.: On existence result for fluids with shear dependent viscosity unsteady flows, In: "Partial Differential Equations", W. Jäger, J. Nečas, O. John, K. Najzar and J. Stará (eds.), Chapman and Hall, 2000, 121-129.

[7] Kreml, O.: Mathematical analysis of models for viscoelastic fluids, Ph.D. thesis, Charles University in Prague, Faculty of Mathematics and Physics, (2010).

[8] Kreml, O.; Pokorný: M.: On the local strong solutions for a system describing the flow of a viscoelastic fluid, Banach Center Publications, Vol. 86: Nonlocal and Abstract Parabolic Equations and their Applications (2009), 196-205.

[9] F. Lin, C. Liu and P. Zhang, On hydrodynamics of viscoelastic fluids, Comm. Pure Appl. Math. 58 (2005), 1437-1471. 
[10] Lions, P.-L., Masmoudi, N.: Global solutions for some Oldroyd models of non-Newtonian flows, Chinese Ann. Math. Ser. B, 21 no. 2 (2000), 131-146.

[11] C. Liu and N. J. Walkington, An Eulerian description of fluids containing visco-elastic particles, Arch. Ration. Mech. Anal. 159 (2001) no. 3, 229-252.

[12] Šalom, P.: Mathematical analysis of a regularized model for visco-elastic nonnewtonian fluid, M.Sc. thesis, Charles University in Prague, Faculty of Mathematics and Physics, (2012).

Ondřej Kreml

Institute of Mathematics of the Academy of Sciences of the Czech Republic

Žitná 25

11565 Praha 1

Czech Republic

kreml@math.cas.cz

Milan Pokorný

Charles University in Prague, Faculty of Mathematics and Physics

Mathematical Institute of Charles University

Sokolovská 83

18675 Praha 8

Czech Republic

pokorny@karlin.mff.cuni.cz

Pavel Šalom

Charles University in Prague, Faculty of Mathematics and Physics

Department of Mathematics Education

Sokolovská 8318675 Praha 8

Czech Republic

pavel.salom@gmail.com 